\title{
Does Distance from the Equator Predict Self-Control? Lessons from the Human Penguin Project
}

1. Hans IJzerman, Vrije Universiteit, Amsterdam, Van der Boechorstraat 1, 1081 BT, Amsterdam, the Netherlands; phone: +31 2059 85868; e-mail: h.ijzerman@ gmail.com.

2. Marija V. Čolić, University of Belgrade, Faculty of Sport and Physical Education, Blagoja Parovića 156, 11030 Belgrade, Serbia; phone: +381 113531000 e-mail: mrjcolic@gmail.com.

3. Marie Hennecke, University of Zurich, Department of Psychology, Binzmuehlestrasse 14/6, 8050 Zurich, Switzerland; phone: +41 4463575 08; e-mail: m.hennecke@psychologie.uzh.ch.

4. Youngki Hong, University of California, Santa Barbara, Department of Psychological and Brain Sciences, University of California, Santa Barbara, Santa Barbara, CA USA; email: youngki.hong@psych.ucsb.edu.

5. Chuan-Peng Hu, Tsinghua University, Department of Psychology, Beijing, 100084, P.R.China; phone: +86 15910258380; email: hcp4715@ gmail.com.

6. Jennifer Joy-Gaba, Virginia Commonwealth University, 806 West Franklin Street, PO Box 842018, Virginia Commonwealth University, Richmond, VA USA; email: jjoygaba@vcu.edu.

7. Dušanka Lazarević, University of Belgrade, Faculty of Sport and Physical Education, Blagoja Parovića 156, 11030 Belgrade, Serbia; phone: +381 113531000 e-mail: dusanka.lazarevic@fsfv.bg.ac.rs.

8. Ljiljana B. Lazarević, University of Belgrade, Faculty of Philosophy, Čika Ljubina 1820, 11000 Belgrade, Serbia; phone +381 11 2639119; e-mail: ljiljana.lazarevic@f.bg.ac.rs.

9. Michal Parzuchowski, SWPS University of Social Sciences and Humanities, Campus in Sopot, ul. Polna 16/20; 81-745, Sopot, Poland.

10. Kyle G. Ratner, University of California, Santa Barbara, Department of Psychological and Brain Sciences, University of California, Santa Barbara, Santa Barbara, CA USA; email: kyle.ratner@psych.ucsb.edu.

11. Thomas Schubert, University of Oslo, Department of Psychology, Postboks 1094, Blindern, 0317 Oslo, Norway; e-mail: thomas.schubert@ psykologi.uio.no.

12. Astrid Schütz, Otto-Friedrich-Universität Bamberg, Fakultät Humanwissenschaften, Markusplatz 3, D-96045 Bamberg, Germany; phone +49 (0) 951 / 863-1870; e-mail: astrid.schuetz@uni-bamberg.de.

13. Darko Stojilović, University of Belgrade, Faculty of Philosophy, Čika Ljubina 18-20, 11000 Belgrade, Serbia; phone +381 11 2639119; e-mail: darkostojilovic@ gmail.com

14. Sophia C. Weissgerber, Universität Kassel, Institut für Psychology, Holländische Str. 3638, 34127 Kassel, Germany; phone +49 561804 7756; e-mail: scweissgerber@ unikassel.de.

15. Janis Zickfeld, University of Oslo, Department of Psychology, Postboks 1094, Blindern, 0317 Oslo, Norway; phone +47 45564234; e-mail: j.h.zickfeld@ @sykologi.uio.no.

16. Siegwart Lindenberg, Department of Sociology (ICS), University of Groningen, Grote Rozenstraat 31,9712 TG Groningen and Tilburg Institute for Behavioral Economics Research (TIBER), Tilburg University, the Netherlands; phone +31 50-3636259; e-mail: s.m.lindenberg@rug.nl. 
This commentary has now been published and can be cited as IJzerman, H., Colic, M. V., Hennecke, M., Hong, Y., Hu, C. P., Joy-Gaba, J., ... \& Lindenberg, S. (2017). Does distance from the equator predict self-control? Lessons from the Human Penguin Project. Behavioral and Brain Sciences, 40. 


\begin{abstract}
We comment on the proposition "that lower temperatures and especially greater seasonal variation in temperature calls for individuals and societies to adopt... a greater degree of selfcontrol", for which we cannot find empirical support in a large dataset with data-driven analyses. After providing more nuance in our theoretical review, we suggest revisiting their model with an eye to the social determinants of self-control.
\end{abstract}

Word count abstract: 62

Word count main text: 986

Word count references: 360

Word count entire text: 1863

Author note: Hans IJzerman is the lead author of this article and Siegwart Lindenberg last. All other authors are listed alphabetically. To whom correspondence should be addressed:

h.ijzerman@gmail.com. 
Van Lange et al. (in press) formulated a theoretical model where they proposed climate as a predictor of self-control (and aggressive behavior). We comment on the proposition "that lower temperatures and especially greater seasonal variation in temperature calls for individuals and societies to adopt... greater degree of self-control", which they argue to be due to a slower life history strategy. In developing their theoretical position, the authors propose distance from the equator as a predictor for self-control. They advocated a "data-driven" approach, "allowing one to derive precise estimates of the variance accounted for by various predictor variables". In our Human Penguin Project (HPP; https://osf.io/2rm5b/), we collected latitude, self-control, and a variety of important social predictors from 14 countries with varying distances from the equator. These variables allowed us to test the proposed relationship between equator distance and self-control. The social predictors included variables such as social network quality and size, and variables that are crucial for life history theory, like people's attachment styles (Del Guidice, 2008). Altogether, we provide the very first test of the authors' proposed model through datadriven analyses (a method called supervised machine learning; for more technical discussions see Breiman, 2001; IJzerman et al., 2016; Yarkoni \& Westfall, 2016) as proposed by the authors and in a more traditional NHST confirmatory manner (a mediation analysis).

We analyzed data from 1537 participants from 14 countries on three different continents, with countries at varying levels of distance from the equator. The underlying analytical details (including a detailed explanation of supervised machine learning) are reported online (https://osf.io/gtj38/). Our method is very powerful and robust, as it validates the model internally as it tests the strength of the model and the size of the error. We found distance from the equator to be a significant predictor of self-control (Tangney et al., 2004), but barely so: It was the 14th predictor in our list, and comparable in prediction power to whether participants 
spoke Serbian or not (the 13th predictor). ${ }^{1}$ As we could compare the strength of different predictors, our analyses revealed that the power to predict self-control was much greater for such variables as attachment anxiety, proneness to feeling nostalgia (a complex social emotion), social network size, the level of complex social integration, and participants' attachments to their homes. Plotting these variables controlling for the remainder of the model further confirmed the stronger relationship of social determinants over distance from the equator (https://osf.io/vzwbe/).

Was distance from the equator then a reliable predictor of climate? We think so: Equator distance correlated strongly with the minimum temperature of that day $(r=-.90, N=1463)$. We further explored whether attachment anxiety would mediate the relationship between equator distance and self-control; attachment anxiety (a strong predictor of self-control) could be indicative of differing life history strategies (Del Guidice, 2008). There was no such relationship, as distance from the equator failed to be related to attachment anxiety $(t=.02, p=.99)$, with a nonsignificant mediation onto self-control (CI95: -.0007-.0007). To be sure, we also tested for attachment avoidance, which also was not predicted by equator distance $(t=-.02, p=.85$; CI95: .0003-.0004). We thus find little evidence that equator distance matters for predicting selfcontrol and life history strategies, and our analyses support the idea that the social environment is much more important in predicting self-control.

How could this be so? The authors reviewed evidence that seemed supportive of their relationship between climate and self-control. However, our reading of the literature suggests more nuance. Warmer water temperatures are indeed associated with faster growth, earlier death, and higher risk acceptance for some animals (all indicative of a faster life history trajectory; Holt

\footnotetext{
${ }^{1}$ Notably, when testing solely for the correlation between equator distance and self-control we find a significant correlation $(r=.12, N=1484)$. However, our machine learning approach did not detect a similar pattern, and we think the correlation is spurious and overfitted (Yarkoni \& Westfall, 2016).
} 
\& Jorgensen, 2014). But in contrast, the warm-blooded Mesopelagic fish (that lives in an extremely cold environment) also shows a precocious maturation comparable to that with other animals in warmer environments (Miya \& Nemoto, 1986). And life history strategies cannot be easily extended from animals to humans. Humans are more unusual in that both slow and fast life history strategies can involve effortful control and impulsivity (Del Giudice, 2015). Furthermore, warmer temperatures are not just related to aggression; a wealth of evidence in humans shows that higher temperatures can also relate to prosocial behavior (cf., IJzerman et al., 2015).

Furthermore, the authors neglected the fact that self-control via internalized norms is not linked to time horizon but to a feeling of obligation. It is thus strongly influenced by the relevance of goals (Lindenberg, 2013). For example, Dutch males scoring higher on honor concerns respond more aggressively when insulted, but less aggressive when not insulted (IJzerman et al., 2007). A recent study also showed that the relationship between armed conflict and heat waves or droughts is due to the fact that heat waves or droughts exacerbate existing ethnic strife (Schleussner et al., 2016). Goal relevance rather than a lack of self-control strengthens aggressive responding in these cases.

In short, a broader consideration of the literature speaks against most of the propositions of their theoretical model. Most critically, our data was suited for testing many of their claims and does not provide empirical support, likely because their theoretical model is underfitted, with the link between climate and self-control being underspecified. It is thus not surprising that the HPP provided evidence against their model, which we take as a strong suggestion for the authors to revisit their proposed link between climate and self-control. 
In reformulating their model, we further advise that they give greater weight to established theories on the complex relationships between self-control, life-histories, culture, social organization and violence, such as the literature mentioned above as well as literature on the development of culture (Diamond, 1999) and the antecedents of violence (Fiske \& Rai, 2015). These theories support our results that the social environment rather than climate predicts self-control and we suspect it is this social environment that mostly predicts aggressive behaviors. Cultures are not individual writ large; they invariantly are complex and cannot be reduced to a simple main-effect model. 


\section{References}

Breiman, L. (2001). Random forests. Machine Learning, 45, 5-32.

Giudice, M. (2008). Sex-biased ratio of avoidant/ambivalent attachment in middle childhood. British Journal of Developmental Psychology, 26, 369-379.

Del Giudice, M. (2015). Self-Regulation in an Evolutionary Perspective (pp.25-41). In G. H. E. Gendolla, S. Koole \& M. Tops (Eds.) Handbook of Biobehavioral Approaches to Self-Regulation. New York: Springer.

Diamond, J. (1999). Guns, Germs, and Steel: The Fates of Human Societies. New York: WW Norton \& Company.

Fiske, A. P. Rai. TS (2015). Virtuous Violence: Hurting and killing to Create, Sustain, End, and Honour Social Relationships. Cambridge University Press.

Holt, R. E., \& Jørgensen, C. (2014). Climate warming causes life-history evolution in a model for Atlantic cod (Gadus morhua). Conservation Physiology, 2, cou050.

IJzerman, H., van Dijk, W. W., \& Gallucci, M. (2007). A bumpy train ride: a field experiment on insult, honor, and emotional reactions. Emotion, 7, 869.

IJzerman, H., Coan, J. A., Wagemans, F., Missler, M. A., Beest, I. V., Lindenberg, S., \& Tops, M. (2015). A theory of social thermoregulation in human primates. Frontiers in Psychology, 6, 464.

IJzerman, H., Pollet, T., Ebersole, C., \& Kun, D. (2016). What predicts Stroop performance? A conditional random forest approach. Available at SSRN: papers.ssrn.com/sol3/papers.cfm?abstract_id=2805205.

Lindenberg, S. (2013). Social rationality, self-regulation and well-being: The regulatory significance of needs, goals, and the self (pp.72-112). In: R. Wittek, T.A.B. Snijders, and 
V. Nee (Eds.), Handbook of Rational Choice Social Research. Stanford: Stanford University Press.

Miya, M., \& Nemoto, T. (1986). Life history and vertical distribution of the mesopelagic fish Cyclothone alba (family Gonostomatidae) in Sagami Bay, Central Japan. Deep Sea Research Part A. Oceanographic Research Papers, 33, 1053-1068.

Schleussner, C. F., Donges, J. F., Donner, R. V., \& Schellnhuber, H. J. (2016). Armed-conflict risks enhanced by climate-related disasters in ethnically fractionalized countries. Proceedings of the National Academy of Sciences, 201601611.

Tangney, J. P., Baumeister, R. F., \& Boone, A. L. (2004). High self-control predicts good adjustment, less pathology, better grades, and interpersonal success. Journal of Personality, 72, 271-324.

Yarkoni, T., \& Westfall, J. (2016). Choosing prediction over explanation in psychology: Lessons from machine learning. Retrieved from http://jakewestfall.org/publications/Yarkoni_Westfall_choosing_prediction.pdf. 